\title{
El voluntariado juvenil y los debates sobre la educación ciudadana. Alemania Occidental y Gran Bretaña en los años cincuenta y sesenta del siglo XX
}

Youths' Volunteering and the Debates about Civic Education. West Germany and Great Britain in the 1950s and 60s

\author{
Christine Krüger \\ Justus-Liebig Universität Gießen \\ christine.krueger@geschichte.uni-giessen.de
}

\begin{abstract}
Resumen
En México, como en muchos otros países del mundo, numerosas iniciativas estatales y no gubernamentales se han dedicado al fortalecimiento de la sociedad civil en los últimos años -y muchos de ellas particularmente a través de la participación de los jóvenes (p. ej. Centro Mexicano para la Filantropía, 2014) - . Políticos, periodistas y científicos promocionan el voluntariado como medida para mejorar la cohesión social y para fomentar la democracia (Aguilar Valenzuela, 2006). Esa valoración es parte de una tendencia global que se refleja por ejemplo en la atención de Naciones Unidas respecto del voluntariado (United Nations General Assembly, 2001). Sin duda alguna, la democracia depende de la participación activa de los ciudadanos. No obstante, el discurso actual normalmente trata el voluntariado y la sociedad civil como instituciones a-históricas e ignora su volubilidad. Con el enfoque en Alemania y Gran Bretaña, dos países en los que los esfuerzos para promover el voluntariado juvenil se efectuaron ya relativamente temprano, ese artículo tiene el objetivo de mostrar que es importante de no entender la sociedad civil y el voluntariado desde una perspectiva normativa pero de considerarles en su historicidad.
\end{abstract}

Palabras clave: sociedad civil, voluntariado, juventud, roles de género, Alemania, Gran Bretaña.

\section{Abstract}

In Mexico like in many other countries of the world many statutory or nongovernmental initiatives have been launched to strengthen civil society in recent years. Many of them have payed particular attention to the involvement of the youth. (e.g. Centro Mexicano para la Filantropía, 2014). Politicians, journalists and social scientists celebrated voluntary work as a means to strengthen social cohesion and to 
foster democracy (Aguilar Valenzuela, 2006). This appreciation is part of a global trend which is reflected, for example, in the interest in voluntary action displayed by the United Nations (United Nations General Assembly, 2001). Undoubtedly, democracy depends on active citizens' participation. However, the recent discourse, normally conceives voluntary action and civil society as historical institutions and ignores their changeability. Focusing on Germany and Britain, two countries, that were forerunners in the promotion of youth' volunteering, this article argues that it is important not to understand civil society and voluntary work in normative terms but to consider them in their historicity.

Keywords: civil society, voluntary action, youth, gender roles, Germany, Great Britain.

\section{Introducción}

Entre 1954 y 1961 se establecieron varios programas de voluntariado juvenil en Alemania y Gran Bretaña. Estos servicios voluntarios tenían el objetivo explícito de crear una nueva institución educativa que promoviera el espíritu comunitario de los adolescentes. Reclutaron jóvenes entre 18 y 25 años de edad para los trabajos voluntarios en el sector social. "Voluntario", en este contexto, significa que los jóvenes no participaron ni como resultado de una obligación estatal, ni para ganarse la vida. En general, estos servicios se realizaron en forma de un trabajo de tiempo completo en una institución social durante un periodo de algunos meses. A cambio los participantes obtuvieron un pago mínimo. Así en los dos países había nacido una institución que se convirtió rápidamente en un gran éxito: desde los comienzos, cientos de miles de jóvenes se sumaron a estas iniciativas.

Los programas británicos y alemanes surgieron de manera independiente y en algunos puntos existen grandes diferencias nacionales. Sobre todo, sus fundadores explicaron de manera distinta su necesidad de emprender el voluntariado. No obstante, también tenían algunas ideas fundamentales en común: en ambos países los fundadores estaban convencidos de que el establecimiento y el crecimiento del Estado de bienestar había generado la necesidad de crear una nueva institución educativa para enseñar a los jóvenes los valores cívicos y para convertirlos en ciudadanos ejemplares. En su opinión, los nuevos derechos sociales iban de la mano de nuevas responsabilidades. Sobre todo en los círculos conservadores, existía un temor generalizado de que en la sociedad del bienestar las antiguas instituciones educativas - la familia, la escuela y el servicio militar - fallaban en la tarea de preparar a los jóvenes para asumir estas nuevas obligaciones. En consecuencia se divulgó la idea de que el Estado o la sociedad civil tenían que proveer programas especiales para compensar esta insuficiencia.

Con el objetivo de estudiar los servicios voluntarios alemanes y británicos, este artículo combina perspectivas comparativas con perspectivas transnacionales para dar atención a las 
diferentes tradiciones nacionales, así como a la influencia recíproca y a los desarrollos comunes. El énfasis en Alemania y Gran Bretaña se explica en primer lugar por la añeja historia de servicios voluntarios para jóvenes en ambos países, y en segundo lugar por las diferencias marcadas entre sus sistemas de bienestar y sus tradiciones de voluntariado, así como de su definición de ciudadanía.

La primera parte de este artículo introduce los debates historiográficos sobre el tema del voluntariado y la sociedad civil. La segunda parte analiza la fundación de los servicios del voluntariado juvenil como reacción al establecimiento del Estado de Bienestar. La tercera parte se dedica al impacto de los roles de género en la concepción del voluntariado juvenil. Finalmente, la última parte trata la transformación paulatina de los servicios debido al proceso de democratización que surge en los años sesenta. El análisis se basa sobre todo en recopilación de datos de las organizaciones patrocinadoras y de archivos estatales -incluyendo cartas, testimonios y memorias de voluntarios- así como en la cobertura de la prensa sobre el tema del voluntariado.

\section{La historiografía sobre el voluntariado}

A pesar del interés público en el tema, en Alemania hay pocos historiadores especializados en el siglo xx en materia del sector voluntario (Kramer/Krüger, 2019). Aquellos que estudian la sociedad civil -y más recientemente al humanitarismo - se concentran muy a menudo en el análisis de los aspectos políticos del tema. Por ejemplo, estudian los movimientos de protesta o el papel de las organizaciones no gubernamentales en las relaciones internacionales, pero descuidan el voluntariado en el ámbito social. Curiosamente, eso es completamente diferente en el Reino Unido. A partir de los años noventa, se ha desarrollado una viva historiografía sobre la acción voluntaria británica, que se institucionaliza de varias maneras (p. ej. Finlayson, 2002; Hilton/McKay, 2011; Oppenheimer/Deakin 2011). Así por ejemplo, se funda la asociación Voluntary Action History Society en 1991, al tiempo que se organizan varias conferencias sobre el tema; y en los últimos años los historiadores británicos no sólo han creado una base de datos sobre archivos dispersos de organizaciones voluntarias, también han establecido un archivo particular que recolecta material sobre la acción voluntaria en el Reino Unido (Voluntary Action History Society, DANGO).

La historiografía británica muestra que el estudio del voluntariado en toda su diversidad arroja luz sobre muchos aspectos del desarrollo sociocultural en la segunda mitad del siglo Xx. A través del voluntariado se pueden analizar los cambios del sistema de bienestar desde una perspectiva que no se limita al desarrollo estatal, lo que facilita considerar en detalle el rol de diferentes actores históricos. Asimismo, ilustra los discursos sobre las fuerzas 
cohesivas de una sociedad y sobre la relación entre el individuo y la sociedad o más específicamente entre el ciudadano y el Estado. Además, el estudio histórico del voluntariado permite verificar las aserciones políticas sobre el funcionamiento de la sociedad civil.

El elogio de los políticos y de los medios de comunicación al voluntariado se basa en el argumento de que una sociedad civil fuerte protege la democracia. Esa idea es antigua y se remonta a Alexis de Tocqueville, en su obra La democracia en América, en donde alaba a la sociedad civil en las asociaciones estadounidenses (Tocqueville, 1835/1840). En la segunda parte del siglo Xx este elogio fue transmitido de las asociaciones al sector voluntario en su conjunto. Se supone que la afiliación a una asociación de la sociedad civil fomenta las reglas democráticas. Esta convicción se consolida sobre todo durante la disolución del antiguo bloque del Este, en cuyo proceso de ruptura se atribuye un papel decisivo a la sociedad civil (Klein, 2007).

Sin embargo, en los últimos años, el vínculo entre sociedad civil y democracia ha sido cuestionado. Sus críticos aceptan que la democracia necesita la participación activa de los ciudadanos, pero objetan que el compromiso voluntario igualmente puede volverse en contra de la democracia y apoyar tendencias antidemocráticas (p. ej. Grande, 2018). Entre ellos, se considera la promoción del voluntariado como medida para encubrir el desmantelamiento del Estado de Bienestar (p. ej. Ruoss, 2019).

\section{La "sociedad adinerada" y la fundación del voluntariado juvenil en los años cincuenta}

Para entender los servicios voluntarios que se crearon en los años cincuenta del siglo xx hay que estudiar sus orígenes más antiguos. Efectivamente, el voluntariado juvenil no era una invención de la segunda mitad del siglo xx. Ya en los años veinte y treinta varios países europeos, así como los Estados Unidos con el famoso Civilian Conservation Corps (ccc), habían introducido servicios laborales para los jóvenes (Schweizer Zentralstelle für freiwilligen Arbeitsdienst and International Student Service, 1938). Algunos de estos servicios eran obligatorios, pero en la mayoría de los casos se reclutaron voluntarios. Ese era el caso en Alemania, cuyo servicio laboral (Freiwilliger Arbeitsdienst) creció rápidamente (Dudek, 1988; Göbel, 2005). En su punto más álgido durante 1932, llegó a incorporar aproximadamente 285000 jóvenes.

Al contrario de los servicios voluntarios que se crearon en los años cincuenta y sesenta, estos servicios laborales sirvieron en primer lugar como medida para reducir el desempleo. Otra diferencia es que los participantes en los servicios laborales normalmente no trabajaron en el sector social pero ayudaron a la construcción de carreteras, en los trabajos forestales o en el cultivo de terrenos baldíos. El modelo inequívoco para los servicios laborales era el 
servicio militar y la idea era que tuviera un complemento. En Alemania - cuyo ejército fue sustancialmente disminuido por el Tratado de Paz de Versalles- mucha gente veía el servicio laboral como sustituto del servicio militar recién suprimido. En 1935, los nacionalsocialistas transformaron este servicio en un servicio obligatorio (Patel, 2005). En Gran Bretaña también se desarrolló un vivo debate público sobre los servicios laborales (labour services o work camps) en el periodo entreguerras. En efecto, en el Reino Unido el Estado así como algunas organizaciones privadas crearon varios servicios voluntarios para jóvenes desempleados durante los años veinte y treinta, pero a menor escala que en Alemania (Field, 2013). Los esquemas estatales se llamaron los Government Training Centres, los Industrial Training Centres y los Training Schemes for Women.

Aunque en una fase de crisis económica el objetivo principal del servicio laboral era combatir el desempleo había igualmente otros motivos para su establecimiento. En esta época, la percepción de un profundo cambio social y la experiencia del paro masivo generaron un amplio sentido de incertidumbre. Las clases medias no solo temieron que el desempleo provocara agitación social, también pensaron que largos periodos de ociosidad conducirían a una decadencia de valores morales. Eso explica por qué durante esta época hubo una inusitada atención a los jóvenes en el discurso público. El pensamiento sobre el futuro se concentró cada vez más en la juventud. Pero, sobre todo, engendró múltiples inquietudes sobre este sector poblacional. Por un lado, los jóvenes eran considerados especialmente idealistas, por otro lado, parecían particularmente amenazados por el cambio social y las vicisitudes de una época turbulenta (Gillis, 1974). La esperanza era que con los servicios laborales la sociedad pudiera beneficiarse del idealismo juvenil, al mismo tiempo que se protegía a los jóvenes de la degeneración moral.

Después de la Segunda Guerra Mundial transcurrió aproximadamente una década hasta que la idea del servicio laboral se reintrodujo y volvió a ganar fuerza. En Alemania una institución de beneficiencia protestante lanzó el primer programa de voluntariado juvenil en 1954. Poco después, otras asociaciones de beneficienca protestantes, católicas y no confesionales introdujeron programas similares. En 1964 el gobierno federal integró estas iniciativas en el Año Social Voluntario (Freiwilliges Soziales Jahr), coordinándolas y financiándolas (Krüger, 2016: 130-132).

En el Reino Unido se fundaron las organizaciones Voluntary Service Overseas en 1958 y la Community Service Volunteers en 1962 (Krüger, 2016: 189-200). La primera pronto empezó a pagar un salario y a reclutar profesionales en vez de voluntarios. La segunda se mantuvo como organización de voluntarios y, aunque no recibió fondos estatales, logró crecer de manera constante.

En ambos países su establecimiento estuvo estrechamente relacionado con las transformaciones sociales y culturales que se efectuaron en esta época. Tuvo lugar en un período de 
bonanza, que en Alemania es caracterizado como de "milagro económico" y que, en Gran Bretaña, el primer ministro Harold Macmillan describió en 1957 con la famosa frase: "a la mayor parte de nuestro pueblo nunca le había ido tan bien" (para el contexto Turner, 1994: 126). ${ }^{1}$ Entre los conservadores, la expansión de la seguridad económica y social generó un pánico moral que se tradujo en un impulso y diseño de los programas de voluntariado en ambos países, aunque en mayor medida en Alemania. De la idea que la sociedad adinerada iba a terminar degenerándose se dedujo la necesidad de crear programas específicos para promover el trabajo no remunerado y superar el materialismo.

Los llamamientos sobre un declive moral coincidían frecuentemente con otra preocupación que surgía en el Estado de Bienestar, es decir, con la presunta destrucción de los patrones familiares tradicionales. El temor era que la nueva generación que estaba creciendo no fuese capaz de desarrollar un espíritu de comunidad al no entrar en contacto con problemas asociados a enfermedades, vejez y asistencia social.2 Por lo tanto, muchos conservadores presionaron para crear una nueva institución educativa para enseñar a los jóvenes los deberes ciudadanos.

La preocupación relacionada con la prosperidad y el bienestar fue un ámbito compartido en Alemania y en Gran Bretaña. No obstante, tenía rasgos diferentes en los dos países. En Alemania se basó en la convicción ampliamente extendida de que la juventud carecía de ideales. Esta visión se refleja, por ejemplo, en el libro influyente de Helmut Schelsky, uno de los sociólogos alemanes más famosos de los años cincuenta y sesenta (Schelsky, 1957). Por el contrario, mucha gente en el Reino Unido creía que los jóvenes eran naturalmente idealistas. Esta convicción se refleja, por ejemplo, en la obra de teatro exitosa de John Osborne Look back in Anger (Osborne, 1956).

\section{Los roles de género y el voluntariado juvenil}

Las imágenes divergentes de los jóvenes que se observaban en los debates sobre el voluntariado estaban estrechamente conectadas con otra diferencia marcada entre los programas de ambos países. Esto es, que los grupos destinatarios eran altamente diferentes. Cuando los comentaristas británicos hablaban del idealismo juvenil, normalmente pensaban sobre todo en los varones. Imaginaban que la juventud masculina era nostálgica del heroísmo del

1 "Most of our people have never had it so good".

${ }^{2}$ Cf. por ejemplo Bundesarchiv (BA), Koblenz, B 189/5784, Entstehung und Bedeutung Freiwilliger Sozialer Dienste, Das Freiwillige Soziale Jahr, 1, Freiwillige Soziale Dienste, Konferenz mit den Programmdirektoren der Rundfunkanstalten am 13. Mai 1966 in Bonn.; BA, Koblenz, B 153/1478, 3/037, Freiwilliger Sozialer Dienst der Jugend, Kurzfassung, 29 de enero 1964. Bdna, 005 Jugendbericht, 1964/65. 
pasado. Alec Dickson, el fundador de la organización Community Service Volunteers, enfatizó una y otra vez que el mayor peligro para el Estado de Bienestar no era la pobreza, sino el aburrimiento: en su opinión, a los jóvenes les faltaban desafíos reales. Pensó que la sociedad de consumo había "castrado su sentido de virilidad" (Dickson, 1976: 84). ${ }^{3}$ Observó dos razones principales para este vacío: Primero, cada vez menos jóvenes pudieron salir de Gran Bretaña para realizar un periodo de servicio público en las colonias. Tal periodo de colonial service tradicionalmente había sido un paso profesional previo a la carrera de funcionario público en Inglaterra. En segundo lugar, el servicio militar obligatorio, que se había introducido en el Reino Unido durante la Segunda Guerra Mundial, terminó en 1960 (anónimo, 1959).

En consecuencia, Dickson concibió el voluntariado juvenil como una "aventura". Su primera organización, la organización de ayuda al desarrollo Voluntary Service Overseas, cumplió con su objetivo enviando jóvenes a países lejanos considerados exóticos. La idea era que los participantes pudieran tener nuevas andanzas sin empañar aún más la reputación de la antigua potencia colonial. Esta reputación había empeorado considerablemente a consecuencia de la guerra de Suez en 1956, que había sido condenada por las Naciones Unidas y que se había convertido en un fiasco para el Reino Unido.

La concepción del servicio como aventura caracterizó sobre todo los primeros años del Voluntary Service Overseas, cuando reclutaba a jóvenes que recién habían terminado su formación escolar y cuya edad oscilaba entre los 17 y 18 años. Sin embargo, comenzó a perder importancia a partir de 1962 cuando la dirección de la organización decidió contratar a profesionales asalariados (Bird, 1998: 39-40). Ese cambio de rumbo fue adoptado a espaldas de Dickson mientras realizaba un viaje en los Estados Unidos. La respuesta de Dickson a esta decisión contraria a su voluntad, supuso su renuncia a la organización, lo que lo llevó a fundar la Community Service Volunteers. Esta nueva organización mantuvo la promoción del voluntariado como una aventura (p. ej. Dickson, 2004: 253-254). ${ }^{4}$

En el Reino Unido los programas de servicio voluntario nacieron basados en el ideal masculino tradicional, aunque también estaban abiertos a la inclusión de mujeres. Crearon oportunidades para ellas que rompieron con los roles de género convencionales. Ciertamente, al principio, la Community Service Volunteers colocaba a los varones en instituciones correccionales, mientras que a las mujeres las enviaba a orfanatos y guarderías infantiles. ${ }^{5}$ Sin embargo, a mediados de los años sesenta, la organización adoptó el objetivo de invertir el

\footnotetext{
${ }^{3}$ Cf. Archives of Volunteering Matters (AVMA), Londres, AGD/Bl/11 Press articles re CSV 1962-9, Dickson, Alec, "More to service than flag days and logs for the old", [recorte de periódico, 1967, fuente desconocida].

${ }^{4}$ Cf. AVM, Londres, ACD/H1/17, Articles re Gap year... voluntary service in West Germany... 1970s, London, Dickson, Alec, A Domestic Development Service for West Germany? [sin fecha]. p. 2; AVM, Londres, AGD/C9/77, papers re united nations study on domestic youth services, reports on different countries including UK volunteer questionnaires, 1972, Dickson, Alec, [manuscrito sin título, sin fecha]. p. 6.

${ }^{5}$ AVM, Londres, ACD/B1/38, Newsletters, 1966-68; Community Service Volunteers. Background Notes, 1965.
} 
reparto tradicional de papeles de género. Para ilustrar cuán positivo podría ser este cambio de rol, Dickson gustaba de describir la inclusión exitosa de una voluntaria en un reformatorio masculino de menores: "Es sorprendente el impacto que una mujer puede tener [en las escuelas de varones]. No es vista tanto como parte del sistema y consigue resultados que nadie más podría lograr" (Bugler, 1965: 20). ${ }^{6}$ En 1967 el informe anual de la organización describía su política de colocar voluntarias en instituciones donde el personal solía ser puramente masculino, al igual que varones en instituciones con personal mayoritariamente femenino: una práctica que denominaba cross posting?

Los defensores de los programas de voluntariado en Gran Bretaña se preocuparon por captar a los jóvenes varones, partiendo de la idea de que, en una sociedad próspera, dichos jóvenes ya no tenían que demostrar su "hombría" en la guerra y la aventura colonial. En Alemania Occidental, donde se había acabado de reintroducir el servicio militar, su atención se dirigió hacia las mujeres. En efecto, ellas representaban el 90 por ciento de los participantes del servicio voluntario. Los conservadores alemanes generalmente combinaron sus críticas al materialismo con una reprimenda contra el número creciente de mujeres empleadas. Temían que las mujeres jóvenes pudieran crecer carentes de espíritu comunitario. Esta preocupación normalmente provenía de una actitud de oposición general contra un cambio de roles de género. Por lo tanto, el año social voluntario tenía también como objetivo atraer a jóvenes mujeres a tareas domésticas. De hecho, desde mediados de los años cincuenta hasta la segunda mitad de los años sesenta, muchos partidarios del voluntariado juvenil lo consideraron como "escuela propedéutica para el matrimonio". ${ }^{8}$

Sin embargo, la impronta conservadora detrás de los programas del voluntariado juvenil no evitó que las participantes utilizaran su servicio como una forma de extender su margen de decisión: muchas participantes ya habían entrado en la vida laboral antes de empezar su voluntariado. La gran mayoría de ellas venían de clase media baja, en donde las mujeres jóvenes seguían sin tener mucha libertad de elección durante esa época (p. ej. anónimo, 1966: 180). Por lo general, no se habían incorporado a su primer trabajo como parte de una elección propia, sino más bien por la decisión de sus padres. Y los padres normalmente las habían enviado a una oficina, bajo la idea de que los trabajos de oficina parecían rentables, estables y adecuados para mujeres. ${ }^{9}$ El trabajo en el sector sanitario, por el contrario, no

\footnotetext{
6 "It is amazing the impact a woman can have [in approved schools for boys]. She's not so much regarded as being part of a set-up and she brings out things that wild horses wouldn't drag."

${ }_{7}$ AVM, Londres, AGD/Bl/39, Community Service Volunteers, Annual Report 1966/67, p. 10

8 Zentralarchiv Neuendettelsau (ZA), Bestand D -Einrichtungen Schulreferat- Diakonisches Jahr, I D5/2-2, Niederfüllbach, 12 de marzo 1955.

${ }^{9}$ Archiv für Diakonie und Entwicklung (ADE), Berlín 4856 Soziales Pflichtjahr für Mädchen, Berlin, Abschrift aus epd ZA Nr. 152, 7 de julio1966:; Waldersee, Etta Gräfin, Grußwort, Deutsches Rotes Kreuz (ed.), Freiwillige Dienste, Bonn 1966. pp. 1-6, p. 3; Archiv der sozialen Demokratie, Bonn, Protokoll der Hauptausschuß-Konferenz vom 12. Dez.
} 
tenía mucho prestigio y era considerado como mal pagado. Sin embargo, numerosas mujeres jóvenes preferían un trabajo asistencial, que les parecía más gratificante. Por lo tanto, muchas empezaron servicios de voluntariado para inducir un cambio de carrera.

Las diferencias entre los servicios voluntarios alemanes y británicos se pueden explicar por tradiciones nacionales. La glorificación de la aventura se basó en los ideales de las escuelas inglesas de élite, que retomaron algunos elementos del movimiento scout (Mechling, 2012; Rosenthal 1986). Por otro lado, el año voluntario alemán retomó con ciertos matices su precursor servicio laboral de la República Alemana de Weimar. Sin embargo, más decisivo que estas tradiciones fueron las experiencias divergentes de los dos países durante la Segunda Guerra Mundial. Esto queda patente al considerar el desarrollo del servicio voluntario de ambos países desde una perspectiva transnacional.

Si nos remontamos al entorno entreguerras observamos que hasta 1933 las ideas sobre los servicios laborales habían diferido poco en ambos países. Muchos británicos habían admirado el servicio laboral alemán, entre ellos Alec Dickson, el fundador de las dos organizaciones de voluntariado británicas. En julio de 1933 Dickson, que entonces era estudiante y tenía 19 años, tuvo la oportunidad de visitar un campo de servicio laboral nacionalsocialista en Alemania. De regreso en Gran Bretaña promovió la idea de los campos laborales en el periódico Yorkshire Post (Dickson, 1933). Mediante ello enfatizaba que el servicio laboral no era "de ninguna manera una innovación del nacionalsocialismo alemán". De igual manera rechazaba la sospecha de que servía para fines militares. En cambio, lo presentaba como una forma útil de contrarrestar la desmoralización de los jóvenes y el conflicto de clases.

Sin embargo, en los años posteriores, la explotación del servicio laboral por parte del nacionalsocialismo con fines de adoctrinamiento y militarización logró empañar la opinión británica sobre esta institución. Ya para el comienzo de la guerra, cualquier posible simpatía subsistente había desaparecido al mismo tiempo que el Estado británico dejó sus esquemas existentes. La historiadora Sonya $O$. Rose demuestra en qué medida la imagen negativa del nacionalsocialismo dio forma a las ideas británicas sobre la ciudadanía (Rose, 2003). Esto también se puede ver en cuanto al debate sobre los servicios voluntarios juveniles.

No obstante, la mera voluntad de diferenciarse del modelo nacionalsocialista no era lo que distinguía al debate británico sobre los servicios voluntarios del debate alemán del período de posguerra. La diferencia entre ambos discursos reside más bien en la forma distintiva en que se definían las características negativas de este contramodelo, como lo muestra el ejemplo de la definición de los roles de género durante posguerra. En esos años se organizaron muchos campamentos internacionales de vacaciones (work camps) para jóvenes, que

1964 in Bonn, p. 10, Berichte der Bezirksverbände über ihre Vorbereitungen zur Durchführung des freiwilligen sozialen Jahres. 
tenían algunos rasgos en común con los servicios voluntarios tratados en este artículo, pero perseguían el objetivo de contribuir a la reconstrucción y a la reconciliación. Eran un lugar de encuentro para jóvenes alemanes y británicos y por esta razón permiten captar de manera particular las peculiaridades de los dos países. Así ilustran que en Gran Bretaña, la distinción tradicional entre trabajos masculinos o femeninos podía estar asociada con la ideología nazi. Por ejemplo, dos jóvenes británicas que participaron en un campamento en Alemania Occidental durante 1948 mostraron su inconformidad al señalar que "ila visión de la Hausfrau (el ama de casa), no se muere en el pensamiento alemán! Mientras los chicos hacen obras de construcción, las chicas cocinan, limpian las mesas y lavan la ropa" . ${ }^{10}$ Sin duda, los roles de género tradicionalmente femeninos seguían siendo persistentes también en Gran Bretaña, pero para estas dos jóvenes eran menos rígidos que en Alemania.

En Alemania no se solía asociar el nacionalsocialismo con los roles tradicionalmente femeninos. Aquí el esfuerzo de diferenciarse del pasado tenía otro efecto en los roles de género. Es decir, que el efecto era sobre los roles masculinos. Al contrario de Gran Bretaña, el elogio del heroísmo y de la masculinidad militar eran un tabú en el discurso público alemán. Por ejemplo, para los servicios de desarrollo alemanes, la idea de que el espíritu de aventura podría ser un motivo adecuado para la participación encontró un amplio rechazo en el público (Maß, 2006: 82-83)." Algunos aspectos que durante mucho tiempo habían pertenecido a la definición común de masculinidad habían desaparecido abruptamente en Alemania occidental después de 1945. Se puede argumentar que esto intensificó los esfuerzos conservadores para insistir en las definiciones tradicionales de femineidad.

El argumento aquí no es que los modelos británico y alemán de voluntariado juvenil se desarrollaran teleológicamente según un patrón único de desarrollo nacional posible. Sin embargo, hay que constatar que los programas evolucionaron con algunas características nacionales muy específicas, que no hubieran sido concebibles en uno y otro país.

\section{La transformación del voluntariado juvenil debido a la democratización de los años sesenta}

La imagen pública que también se encuentra en la historiografía es que los años cincuenta y sesenta fueron un período de estancamiento para el sector voluntario (Finlayson, 2002:

\footnotetext{
${ }^{10}$ Library and Archives of the Religious Society of Friends, Londres, FSC/GE/18/2, Friends Service Council, Monthly Report No 5 Youth work.

${ }^{11}$ Evangelisches Zentralarchiv (EZA), Berlín, 97/734, Korrespondenz und Tagebücher von Freiwilligen in Israel 1961-64, Carta de Johannes Müller a Lothar Kreyssig, Joure, 5 de junio 1961; EZA, Berlín, 97/932, Carta de K. P. a Lothar Kreyssig, 12 de octubre 1965. Lothar Kreyssig, Korrespondenz mit Freiwilligen, 1964/65.
} 
capítulo 4). Sin embargo, en los últimos años, varios historiadores han contradicho esa imagen (Hilton/McKay, 2011). Suponen que la ampliación de los sistemas de bienestar no resultó en un descenso del trabajo voluntario, sino más bien en un cambio de sus campos de aplicación. El establecimiento de servicios de voluntariado juvenil confirma esta argumentación. En Alemania como en Gran Bretaña, el voluntariado juvenil nació no en un período de crisis del Estado de Bienestar, sino en su época de oro. Es importante hacer notar que se creó no para sustituir la prestación pública de servicios sociales sino para complementarla. En este punto había por entonces un consenso político que unía la derecha y la izquierda. En efecto, la existencia del Estado de bienestar reconcilió a la izquierda con el concepto del voluntariado. Tradicionalmente, los partidos socialistas y los sindicatos habían rechazado el trabajo voluntario (Kramer/Krüger 2019; Finlayson, 2002: 11). Lo consideraron como socavación del nivel salarial. Asimismo, calificaron la caridad como paternalismo. En el curso de los años sesenta, sin embargo, empezaron a reconocer el valor del voluntariado como participación ciudadana.

Es importante añadir que los representantes de la izquierda enfatizaron la necesidad del Estado de Bienestar como precondición para que el trabajo voluntario no llegara a condiciones de desigualdad, ya que permitía un compromiso de todas las clases sociales con la acción ciudadana (p. ej. Hasenclever, 1965/1966). No obstante, el consenso sobre la justificación del voluntariado como complemento al Estado de Bienestar se rompió en la larga década de los sesenta. Las fisuras se hicieron en ambas partes del espectro político por varias razones.

Como hemos visto, la introducción de los programas de voluntariado para jóvenes en los años cincuenta y sesenta fue parte de un esfuerzo por restaurar el sistema de valores convencional. La gran mayoría de sus defensores se preocupó por el orden de género, pero no por el orden político. Eso cambió a finales de los años sesenta y a principios de los setenta. Más tarde, en 1968, los objetivos de restauración quedaron anticuados — sobre todo el objetivo de atraer a los jóvenes-. Asimismo, en esos años, los partidarios de la Nueva Izquierda lanzaron fuertes ataques contra el concepto general de trabajo voluntario. Asumieron de nuevo la crítica socialista tradicional y argumentaron que el trabajo voluntario en el ámbito social sólo curaba los síntomas, y por lo tanto estabilizaba el sistema en vez de inducir a un cambio fundamental. ${ }^{12}$ Esta crítica tuvo impactos bastante diferenciados sobre las organizaciones patrocinadoras de servicios voluntarios en Gran Bretaña y Alemania Occidental.

\footnotetext{
12 Por ejemplo ADE, Berlín, ADW HGSt, III 252 Diakonisches Jahr, 2474, Motivanalysen Theodor Schober an die Mitglieder des Diakonischen Rates, 23. Juni 1970, 2. Anlage, Protokoll über ein Grundsatzgespräch (marzo 1970); cf. EZA, Berlín, 97/1841, Beiträge zu Friedensdienst und Kriegsdienstverweigerung, Wolfgang v. Eichborn, Probleme der Friedensdienste. Wandel der Motivation, 1970
} 
En Alemania, los ataques de la Nueva Izquierda llevaron a una crisis del voluntariado juvenil. El número de participantes del Año Social Voluntario se redujo entre 1968 y 1970 por primera vez desde su fundación (Arbeitskreis Freiwilliger Sozialer Dienst/Freiwilliges Soziales Jahr, 1973: 51). Sobre todo los jóvenes con una formación escolar secundaria, que apoyaban en gran medida el movimiento de protesta, se mantuvieron fuera de estos programas. ${ }^{13}$ En consecuencia, las organizaciones proveedoras decidieron cambiar la estrategia publicitaria intentando reorientar sus programas de voluntariado hacia la generación de protesta.

Algunos signos del cambio conceptual ya se habían hecho sentir en el curso de los años sesenta. Pero fue en el nuevo clima político del movimiento estudiantil que varios de los principios básicos del Año Social Voluntario parecían injustificables. Especialmente, se abandonó el objetivo de preparar a las mujeres para el matrimonio. En cambio, las organizaciones voluntarias se apresuraron a modificar su autoconcepto en conformidad con el nuevo clima político. Aunque en principio no cambiaron las labores específicas de los voluntarios en las instituciones sociales, las organizaciones empezaron a presentar su programa como una guía "para cambiar la sociedad" o "para desarrollar un estilo de vida democrático". ${ }^{4}$ Además, elogiaron a los voluntarios porque en su opinión tenían la independencia necesaria para desvelar deficiencias y cuestionar jerarquías (Arbeitskreis freiwilliger sozialer Dienst/Freiwilliges soziales Jahr, 1973: 77). ${ }^{15}$

Por tanto, si bien las tareas de los voluntarios cambiaron poco, el periodo del "movimiento del 68" marcó una clara ruptura en la definición y legitimación del Año Social Voluntario en Alemania. Eso no significa que muchos jóvenes confiaran en esta reorientación. En las fuentes no se encuentran testimonios explícitos sobre este tema. En cualquier caso, el número de participantes no se incrementó significativamente hasta mediados de los setenta, es decir, hasta un momento en que las tensiones del mercado laboral presionaron a muchos jóvenes.

En Gran Bretaña el voluntariado juvenil no enfrentó una crisis similar. El concepto general detrás de la Community Service Volunteers podía adaptarse más fácilmente a las protestas juveniles. Por un lado, esto se debe al hecho de que las protestas, por lo general, fueron menos intensas que en Alemania. Además, desde el comienzo, se había diseñado un programa basado en el modelo tradicional de ciudadanía masculina. Este diseño estaba mucho más cerca del ciudadano políticamente activo, lo cual era el ideal del movimiento de protesta, que en el caso alemán se sustentó en la figura del ama de casa. Por ejemplo, la Commu-

${ }^{13}$ Cf. por ejemplo ADE, Berlín, ADW HGSt, III 252 Diakonisches Jahr, 2474, Motivanalysen Müller-Schöll, Albrecht/ König, Mechthild, Anlage 1: Schriftlicher Bericht über das Diakonische Jahr (Febr. 1970), 9 de febrero 1970.

${ }^{14}$ BA, Koblenz, B 189/5782, 2665-459-(22). Das Freiwillige Soziale Jahr im DPWV, Jahresbericht 1971. p. 9.

${ }^{15}$ Cf. EZA, Berlín, 97/1841 Beiträge zu Friedensdienst und Kriegsdienstverweigerung. Aktionsgemeinschaft Dienst für den Frieden, Rahmenkonzept für die Freiwilligendienste, Eingangsstempel: 12. April 1972. 
nity Service Volunteers siempre había luchado con vehemencia para no ser asociada con la caridad paternalista tradicional. En ese sentido, Dickson declaró el voluntariado como un derecho de todas las clases sociales (anónimo, 1967). Por lo tanto, desde el principio, su organización había invertido un gran esfuerzo por ganarse a los grupos más desfavorecidos de la sociedad y sumarlos a su programa (Orr, 1967). ${ }^{16}$ Por ejemplo, estableció un programa para jóvenes en reformatorios. El objetivo de reclutar a jóvenes de todas las clases sociales claramente puede explicarse por las experiencias que Dickson había tenido en su primera organización: Voluntary Service Overseas. Estaba convencido de que con el idealismo de la juventud, los jóvenes voluntarios podían contribuir a eliminar barreras entre los países colonizados y los países colonizadores (anónimo, 1959; Dickson, 1960: 450; Dickson, 1964: 13; Zinkin, 1962). Como muchos de sus contemporáneos creía que la población de los países colonizados carecía de iniciativa propia y que los jóvenes británicos podían contagiarlos con su entusiasmo. En el contexto de la descolonización, no sorprende que esta concepción fuera pronto rechazada por su grado de prepotencia colonialista (p. ej. Dickson, 1960: 459). Eso también explica por qué los otros miembros de la dirección del Voluntary Service Overseas preferían reclutar profesionales. Por cierto, eso fue también la estrategia del famoso American Peace Corps (Cuerpo de Paz estadounidense) establecido por el presidente Kennedy. El Peace Corps no sólo era una competencia directa para el Voluntany Service Overseas, también marcó la pauta en su época, siendo referencia para el público en general (Wainwright, 1962).

Esto explica por qué Dickson trató de evitar cometer el mismo error con su nueva fundación. No quería que la Community Service Volunteers fuese asociada con un segmento de clase alta actuando por paternalismo o condescendencia. $Y$ aunque un porcentaje elevado de los voluntarios de la organización venía de escuelas de élite, en conjunto, la organización logró reclutar a participantes que por lo general provenían de distintos estratos.

En definitiva, es evidente que la organización pudo convencer a los jóvenes de que su programa de voluntariado compartía sus convicciones y servía para cambiar la sociedad. Como resultado, los números de voluntarios seguían creciendo a finales de los años sesenta. En 1968 la dirección de la organización notó con satisfacción un efecto de reclutamiento de muchos estudiantes de la London School of Economics, los cuales eran conocidos por su inclinación a favor del movimiento estudiantil (anónimo, 1968).

Se ve que muchas diferencias persistieron entre los servicios voluntarios británicos y alemanes. Sin embargo, al mismo tiempo también se pueden observar convergencias que se explican por el carácter transnacional del movimiento de 1968, así como de las reacciones que suscitaron. Parece obvio que la crítica de la Nueva Izquierda sobre la caridad tradicional

${ }^{16}$ Cf. AVM, Londres, AGD/Cl/68, Press Cuttings 1973-76, June 19th 1975 -Southern General Hospital. S.A.S.D. Da Conference- Community Service and the offender. 
llevó a una disminución en las diferencias conceptuales de los programas del servicio voluntario en ambos países. Así pues, el movimiento estudiantil mostró al público alemán que a los jóvenes no les hacía falta el idealismo y que estaban dispuestos a involucrarse. ${ }^{17}$ Sin embargo, los partidarios más conservadores del voluntariado se mantuvieron convencidos de que se necesitaba una institución de formación de jóvenes, que los llevara a ser buenos ciudadanos. Debido al intercambio transnacional creciente al nivel europeo, sus esfuerzos también promovieron la adecuación de los servicios alemanes y británicos. Frente al terrorismo emergente muchos alemanes sentían la necesidad de encontrar una salida constructiva para el impulso revolucionario de la juventud. En este aspecto, la Community Service Volunteers apareció como un modelo por su tradicional compromiso por ofrecer posibilidades de emancipación y aventura a los jóvenes. En 1977 el presidente alemán Walter Scheel invitó a Dickson a discutir sobre cómo la voluntad de cambio de los jóvenes podría ser canalizada de una manera que no condujera al terrorismo.18

Por otro lado, los jóvenes en Alemania que participaron en el movimiento de protesta consideraron que las organizaciones británicas se ajustaban demasiado "al sistema" —como se dijo en esta época - Por ejemplo, un voluntario alemán que hacia su servicio en el Reino Unido criticó la falta de conciencia política de los voluntarios de la Comunity Service Volunteers. En su opinión, simplemente estaban remplazando a los trabajadores remunerados sin ir en contra de los abusos sociales, dificultando con ello el "establecimiento de una conciencia de clase". 19

En resumen, se puede constatar que la crítica de la Nueva Izquierda sacudió el pensamiento sobre el voluntariado en Alemania, mucho más que en Gran Bretaña. Además cabe resaltar que el impacto de las ideas de la Nueva Izquierda fue un elemento decisivo para el desarrollo de los servicios voluntarios durante la crisis económica en los setentas.

\footnotetext{
${ }^{17}$ ADE, Berlín, 4824, ADW, hgst, III 251, Jahr für die Kirche Aktennotiz Gespräch, Diakonisches Jahr-Jahr für den Nächsten am 21. Juni 1968 in Bonn-Venusberg, Liebfrauenhaus, 9-15 Uhr.

${ }^{18}$ Eso fue la interpretación de Dickson, Alec: AVM, Londres, AGD/Cl/9. Annual Reports 1968-80. Annual Report, $1977 / 78$, p. 2. Un breve informe sobre la visita de Dickson en el archivo del Bundespräsidialamt no confirma pero tampoco contradice el relato de Dickson, BA, Koblenz, B 122/23876, Bundespräsidialamt.

${ }^{19}$ EZA, Berlín, 97/494, Großbritannien, Briefe von Freiwilligen Carta de un voluntario, lona, 14/15 de agosto 1976; similar: Carta de un voluntario, 22 de julio 1976, ambos documentos.
} 


\section{Conclusión}

El análisis del establecimiento de los servicios voluntarios para jóvenes en Alemania y Gran Bretaña muestra que el desarrollo del voluntariado ha sido relacionado más con la constitución social que con el ordenamiento político. Incluso muestra que el voluntariado no es necesariamente un signo de la democracia pero igualmente podía ser la consecuencia de temores frente a las tendencias de democratización social que se manifestaron en la expansión del sistema de bienestar así como en la creciente igualdad de género.

Asimismo, la historia de los servicios voluntarios para jóvenes igualmente muestra que los esfuerzos de promover el voluntariado tampoco se explican exclusivamente por la crisis percibida del Estado de Bienestar y por el auge del neoliberalismo. El hecho de que el voluntariado juvenil es un producto de la fase de oro del Estado de Bienestar refuta la ecuación simple de que a mayor Estado de Bienestar, mayor reducción del sector voluntario.

La motivación central para el establecimiento de los servicios voluntarios para jóvenes era el objetivo de crear una nueva institución educativa. La idea era que los nuevos derechos en la sociedad de bienestar tenían que ir de la mano de nuevos deberes. Y para enseñar esos deberes los fundadores del voluntariado juvenil consideraron inadecuadas las instituciones tradicionales - como la familia o el militar-. De esa idea se dedujo la necesidad de una nueva institución para enseñar los valores comunitarios.

\section{Fuentes}

Archivos

Archiv für Diakonie und Entwicklung, Berlín:

Fondo: ADW, HCSt, III 251.

Fondo: ADW, HCSt, III 252

Archiv der sozialen Demokratie:

Fondo: Berichte der Bezirksverbände über ihre Vorbereitungen zur Durchführung des freiwilligen sozialen Jahres.

Archives of Volunteering Matters, Londres:

Fondo: $\mathrm{ACD} / \mathrm{Bl} / 11$

Fondo: $A G D / B 1 / 38$

Fondo: $\mathrm{AGD} / \mathrm{Bl} / 39$.

Fondo: $\mathrm{ACD} / \mathrm{Cl} / 68$.

Fondo: $\mathrm{ACD} / \mathrm{Cl} / 9$.

Fondo: ACD/C9/77.

Fondo: $\mathrm{ACD} / \mathrm{Hl} / 17$.

Bundesarchiv, Koblenz:

Fondo: B 153/1478, 3/037.

Fondo: B 189/5782. 
Fondo: B 189/5784.

Fondo: B 122/23876.

Evangelisches Zentralarchiv, Berlín:

Fondo: 97/1841.

Fondo: $97 / 494$.

Fondo: $97 / 734$.

Fondo: 97/932.

Library and Archives of the Religious Society of Friends, Londres:

Fondo: FSC/CE/18/2.

Zentralarchiv, Neuendettelsau:

Fondo: D5/2-13.

Fondo: D5/2-2.

Fondo: D5/2-28.

Bibliografía

Aguilar Valenzuela, Rubén (2006), "Las organizaciones de la sociedad civil en México: Su evolución y principales retos", tesis doctoral, Universidad Iberoamericana, México.

Anónimo (1959), "Schoolboy Recruits for Central Africa", The Manchester Guardian, 4 de abril.

Anónimo (1966), "Freiwilliges Soziales Jahr. Berichte der Helferinnen", Tübinger Brief, vol. 13, núm. 7, pp. 177-181.

Anónimo (1967), "Dickson’s private army in shackles", Western Mail, 29 de septiembre.

Anónimo (1968), "The $£ 2$ student revolution", The Guardian, 2 de junio.

Arbeitskreis Freiwilliger Sozialer Dienst/Freiwilliges Soziales Jahr (coords.) (1973), Das freiwillige soziale Jahr, Ein dokumentarischer Bericht, Bonn.

Benz, Wolfgang (1968), "Vom Freiwilligen Arbeitsdienst zur Arbeitsdienstpflicht", Vierteljahrshefte für Zeitgeschichte, vol. 16, núm. 4, pp. 317-346.

Bugler, Jeremy (1965), "The Volunteer Upsurge", New Society, 14 de octubre.

Centro Mexicano para la Filantropía (2014), Guía para planificar, crear y fortalecer una organización de la sociedad civil, Cemefi, México.

Dickson, Alec (1933), "Germany's way with the unemployed. British Visitor's Experiences in a Work Camp for Youth", Yorkshire Post, 28 de septiembre.

Dickson, Alec (1960), "Voluntary Service Overseas", Journal of the Royal Society of Arts, mayo, pp. 443-460.

Dickson, Alec (1976), A Chance to Serve, Dennis Dobson, Londres.

Dickson, Mora (1964), A World elsewhere. VSO, Dennis Dobson, Londres.

Dickson, Mora (2004), Portrait of a Partnership, Rovertson Publications, Aberfeldy.

Dudek, Peter (1988), Erziehung durch Arbeit. Arbeitslagerbewegung und freiwilliger Arbeitsdienst 1920-1935, Westdeutscher Verlag, Opladen.

Field, John (2013), Working Men's Bodies. Work Camps in Britain, 1880-1939, Manchester 2013, Manchester University Press, Manchester.

Finlayson, Geoffrey (2002), Citizen, State, and Social Welfare in Britain 1830-1990, Clarendon Press, Oxford.

Grande, Edgar (2018), "Zivilgesellschaft, politischer Konflikt und soziale Bewegungen", Forschungsjournal Soziale Bewegungen, vol. 31, pp. 52-60.

Hasenclever, Christa (1965/66), "Die Bedeutung der Mitarbeit des Staatsbürgers und der freiwilligen Sozialarbeit in einer modernen Gesellschaft", Jahrbuch der Arbeiterwohlfahrt, pp. 67-80.

Hilton, Matthew y McKay, James (coords.) (2011), The Ages of Voluntarism. How We Got to the Big Society, Oxford University Press, Oxford. 
Illian, Christian (2005), Der Evangelische Arbeitsdienst: Krisenprojekt zwischen Weimarer Demokratie und NS-Diktatur. Ein Beitrag zur Geschichte des Sozialen Protestantismus, Gütersloher Verlags-Haus, Gütersloh.

Klein, Ansgar (2001), Der Diskurs der Zivilgesellschaft. Politische Kontexte und demokratietheoretische Bezüge der neueren Begriffsverwendung, Leske + Buderich, Opladen.

Köhler, Henning (1976), Arbeitsdienst in Deutschland: Pläne und Verwirklichungsformen bis zur Einführung der Arbeitsdienstpflicht im Jahre 1935, Duncker \& Humblot, Berlin.

Kramer, Nicole y Krüger, Christine G. (2019), "Einleitung" en Kramer, Nicole y Krüger, Christine (coords.), "Freiwilligenarbeit und gemeinnützige Organisationen im Wandel. Neue Perspektiven auf die Geschichte des 19. und 20. Jahrhunderts", Historische Zeitschrift. Beiheft, núm. 76, pp. 9-30.

Krüger, Christine G. (2016), Dienstethos - Abenteuerlust- Bürgerpflicht. Jugendfreiwilligendienste in Deutschland und Großbritannien im 20. Jahrhundert, Vandenhoeck \& Ruprecht, Göttingen.

Maß, Sandra (2006), "'Eine Art sublimierter Tarzan'. Die Ausbildung deutscher Entwicklungshelfer und helferinnen als Menschentechniker in den 60er Jahren", Werkstatt Geschichte, vol. 42, pp. 77-89.

Mechling, Jay (2012), "Children in Scouting and other Organizations", en Fass, Paula (coords.), Routledge History of Childhood in the Western World, Routledge, Londres.

Oppenheimer, Melanie y Deakin, Nicholas (coords.) (2011), Beveridge and Voluntary Action in Britain and the Wider British World, Manchester University Press, Manchester.

Orr, Majorie (1967), "Using young people in new patterns of community service", The Glasgow Herald, 19 de agosto.

Osborne, John (1956), Look Back in Anger, Evans Brothers, London.

Rose, Sonya O. (2003), Which People's War? National Identity and Citizenship in Britain 1939-1945, Oxford University Press, Oxford.

Rosenthal, Michael (1986), The Character Factory: Baden-Powell and the Origins of the Boy Scout Movement, Pantheon Books, Londres.

Ruoss, Matthias (2019), "Die Neuen Freiwilligen", en Kramer, Nicole y Krüger, Christine G. (coords.), "Freiwilligenarbeit und gemeinnütziger Organisationen im Wandel. Neue Perspektiven auf die Geschichte des 19. und 20. Jahrhunderts", Historische Zeitschrift. Beiheft, núm. 76, pp. 154-180.

Schelsky, Helmut (1957), Die skeptische Generation. Eine Soziologie der deutschen Jugend, Diederichs, Düsseldorf.

Tocqueville, Alexis (1835/1849), De la démocratie en Amérique, 2 vols., Gosselin, Paris.

Turner, John (1994), Macmillan, Longman, London.

Wainwright, David (1962), "Voluntary Service Overseas", The Guardian, 12 de octobre.

Waldersee, Etta Gräfin (1966), "Grußwort”, en Deutsches Rotes Kreuz (coord.), Freiwillige Dienste, Bonn. Zinkin, Taya (1962), "Spirit of youth", The Guardian, 30 de mayo, p. 20.

\section{Recursos electrónicos}

DANGO (Database of Archives of Non-Governmental Organisations), http://www.dango.bham.ac.uk (fecha de consulta: 5/05/2019).

United Nations General Assembly (coords.) (2001), Support for volunteering. Report of the Secretary General, A 56/288, 14 de agosto. http://www.un.org/documents/ga/docs/56/a56288.pdf (fecha de consulta: 5/06/2019).

Voluntary Action History Society, http://www.vahs.org.uk/ (fecha de consulta: 5/06/2019). 
Christine Krücer es profesora en la Universidad de Gießen (Alemania). Es egresada del doctorado en historia en la Universidad de Tübingen en 2005. Ha sido investigadora visitante a la Universidad de Oxford y en SciencesPo Paris. Sus líneas de investigación versan en torno a temas de historia judía, historia del voluntariado e historia urbana. Es autora de Sind wir denn nicht Brüder?" Deutsche Juden im nationalen Krieg, 1870/71 (Schöningh, Paderborn, 2006) y de Dienstethos, Abenteuerlust, Bürgerpflicht. Jugendfreiwilligendienste in Deutschland und Großbritannien im 20. Jahrhundert (Vandenhoeck \& Ruprecht, Göttingen, 2016).

Recibido: 7 de junio de 2019

Aceptado: 25 de junio de 2019 